\title{
BMJ Open Opportunities and challenges to improving antibiotic prescribing practices through a One Health approach: results of a comparative survey of doctors, dentists and veterinarians in Australia
}

Annie Zhuo, ${ }^{1}$ Maurizio Labbate, ${ }^{2,3}$ Jacqueline M Norris, ${ }^{4,5}$ Gwendolyn L Gilbert, ${ }^{5}$ Michael P Ward, ${ }^{4,5}$ Beata V Bajorek, ${ }^{6}$ Chris Degeling, ${ }^{7}$ Samantha J Rowbotham, ${ }^{8,9}$ Angus Dawson, ${ }^{10} \mathrm{Ky}$-Anh Nguyen, ${ }^{11,12}$ Grant A Hill-Cawthorne, ${ }^{5,13}$ Tania C Sorrell, ${ }^{5,14}$ Merran Govendir, ${ }^{4}$ Alison M Kesson, ${ }^{5,15,16}$ Jonathan R Iredell, ${ }^{5,14,17}$ Dale Dominey-Howes ${ }^{1,5}$

To cite: Zhuo A, Labbate M, Norris JM, et al. Opportunities and challenges to improving antibiotic prescribing practices through a One Health approach: results of a comparative survey of doctors, dentists and veterinarians in Australia. BMJ Open 2018;8:e020439. doi:10.1136/ bmjopen-2017-020439

- Prepublication history and additional material for this paper are available online. To view these files, please visit the journal online (http://dx.doi org/10.1136/bmjopen-2017020439).

$\mathrm{ML}$ and DD-H contributed equally.

Received 4 November 2017 Revised 15 January 2018 Accepted 23 February 2018

Check for updates

For numbered affiliations see end of article.

Correspondence to

Associate Professor Maurizio Labbate;

maurizio.labbate@uts.edu.au and Professor Dale DomineyHowes;

dale.dominey-howes@sydney. edu.au

\section{ABSTRACT}

Objectives To explore and compare the knowledge, attitudes and experiences of doctors, dentists and veterinarians (as prescribers) in relation to antibiotic use and antibiotic resistance (AbR), and to consider the implications of these for policy-making that support a One Health approach.

Design A cross-sectional survey conducted online. Setting Doctors, dentists and veterinarians practising in primary, secondary or tertiary care in Australia.

Participants 547 doctors, 380 dentists and 403 veterinarians completed the survey.

Main outcome measures Prescribers' knowledge, attitudes and perceptions of $\mathrm{AbR}$, the extent to which a range of factors are perceived as barriers to appropriate prescribing practices, and perceived helpfulness of potential strategies to improve antibiotic prescribing in practice.

Results There was substantial agreement across prescriber groups that action on $A b R$ is required by multiple sectors and stakeholders. However, prescribers externalised responsibility to some extent by seeing the roles of others as more important than their own in relation to AbR. There were common and context-specific barriers to optimal prescribing across the prescriber groups. Prescriber groups generally perceived restrictive policies as unhelpful to supporting appropriate prescribing in their practice.

Conclusions The results have implications for implementing a One Health approach that involves doctors, dentists and veterinarians as key players to tackling the crisis of AbR. The findings are that (1) prescribers understand and are likely receptive to a One Health policy approach to AbR, (2) policy development should be sensitive to barriers that are specific to individual prescriber groups and (3) the development and introduction of interventions that might be perceived as reducing prescriber autonomy will need to be carefully designed and implemented.

\section{Strengths and limitations of this study}

- The simultaneous surveying of doctors, dentists and veterinarians allowed for direct comparisons between knowledge, attitudes and perceptions to reveal the gaps, opportunities and challenges for a One Health approach towards appropriate the use of antibiotics - a global first.

- The development of the questionnaires was undertaken in partnership with the key national policy-making authority in the Department of Health ensuring relevancy.

- The sample size is modest. However, respondent characteristics were broadly comparable with national workforce statistics, representing a range of practice types and locations, years of experience, age and gender. Some groups in some Australian states were under-represented in the sample.

- The self-selected approach to participant recruitment could have led to selection bias of individuals who were already interested in or knowledgeable about the topic of antibiotic resistance and stewardship.

\section{INTRODUCTION}

Antimicrobial resistance (AMR) is recognised as a complex problem and global threat to human and animal health. ${ }^{1}$ Consequently, the WHO and its member states advocate a One Health approach to tackle the crisis of AMR. ${ }^{2}{ }^{3}$ One Health recognises that the health of humans, animals and the environment is interlinked such that collaborative, multidisciplinary efforts of researchers, policy-makers, 
practitioners and other stakeholders will be required locally, nationally and globally to achieve an effective response. ${ }^{4}$ Due to the complexity of AMR, those tasked with the responsibility of policy-making (which here includes the development of regulations, guidelines, plans, strategies and interventions) require a deeper understanding of the key drivers across the supply and demand sides of antimicrobial use. ${ }^{5}$

A cornerstone of the One Health approach to AMR and a key priority of the WHO AMR response strategy is to reduce misuse of antimicrobials and support optimal prescribing practices in human and animal health. ${ }^{23}$ The effectiveness of strategies that aim to improve prescribing practices depend, in part, on the extent to which factors affecting suboptimal prescribing, barriers to change and prescriber attitudes to potential interventions are understood and addressed. ${ }^{6}$ With this in mind, policies will need to be sensitive to the commonalities and differences between prescriber groups. A complex set of factors influence antimicrobial prescribing behaviour among doctors, dentists and veterinarians. These include the availability and acceptance of guidelines and other information sources; level of knowledge and training; perceptions about the causes, impacts and risks of AMR; availability of diagnostic facilities and diagnostic uncertainty; pressure from patients/clients; fear of clinical failure; time pressures and social and organisational contexts. ${ }^{7-12}$

Empirical research is needed to support targeted, effective policies to change prescribing practices. To date, investigation of knowledge, attitudes, perceptions and behaviours among practitioners has generally involved single professions only. This limits the range and comparability of information needed to support the development of policies that target multiple prescriber groups. ${ }^{13}$ In addition, no surveys have compared prescribers' understanding of the interconnected nature of AMR across human, animal and environmental sectors. Given that One Health is central to international and national efforts to manage AMR, the response of prescribers to this new approach has implications for whether it will be accepted. To address these gaps, concurrent surveys were undertaken of three key prescriber groups in Australia: doctors, dentists and veterinarians. The aims were to: (1) identify commonalities and differences between prescribers' knowledge, attitudes and perceptions of AMR, factors and barriers influencing prescribing and perceptions of possible intervention strategies to improve antibiotic prescribing and (2) consider the implications of the findings for policy-making that support a One Health approach. For the purpose of this study, antibiotic resistance $(\mathrm{AbR})$ is the focus rather than AMR, because antibiotics are the most commonly prescribed antimicrobials. This study uses the WHO definition of 'antibiotic' which is a drug used to prevent and treat bacterial infections. ${ }^{14}$

\section{METHODS}

\section{Study design}

Self-administered surveys targeted doctors, dentists and veterinarians in Australia, canvassing information on demographics, practice characteristics, knowledge, attitudes and beliefs about antibiotic use and effectiveness, barriers influencing prescribing, and attitudes towards possible interventions. Demographic and practice-related questions were profession-specific. Otherwise questions were identical.

Survey questions were designed in collaboration with experienced doctors, dentists and veterinarians, who also assisted with data analysis (see coauthors). Some question items were adapted from existing studies targeting doctors ${ }^{15-17}$ and veterinarians, ${ }^{18} 19$ and qualitative studies exploring knowledge and perceptions among doctors, ${ }^{20}$ dentists $^{8}{ }^{21}$ and veterinarians. ${ }^{9}$ Feedback was received from the Office of Health Protection, The Department of Health, Australian Government, who are coleading Australia's AMR strategy 2015-2019 and implementation plan, to ensure that the data generated were relevant to national policy development. ${ }^{3}$ Most questions were closed-ended with Likert-type responses measured on 4-point or 5-point scale. 'Unsure', 'neutral' and 'not applicable (N/A)' options were also provided where appropriate. The questionnaire was pretested in all three professions and feedback was used to guide the final design.

\section{Recruitment and data collection}

Surveys were hosted on SurveyMonkey and distributed online between October and December 2016. Respondents were reached via researchers' networks, professional associations, professional colleges and practitioner registers through their newsletters, bulletins, email lists, forum announcements and websites (see online supplementary material). For dentists, a marketing mailing list was also used.

\section{Data and statistical analyses}

Statistical analyses were conducted using IBM SPSS Statistics V.22. Respondents completing the demographic questions and at least one other question was included in the analyses. In cases of missing data on a single item, the respondent was excluded from the respective analysis (listwise deletion). Medians and IQRs were calculated and reported for questions with an ordinal response scale. Kruskal-Wallis H statistical tests were conducted to determine whether there were significant $(\mathrm{p}<0.05)$ differences in median responses and mean ranks between doctors, dentists and/or veterinarians (referred to in the notes at the bottom of figures 1-3, respectively); post hoc analyses of pairwise comparisons were performed using Dunn's (1964) procedure, with a Bonferroni correction for multiple comparisons, to estimate adjusted $\mathrm{p}$ values. ${ }^{22} \mathrm{~N} / \mathrm{A}$ ' and 'Unsure' responses were excluded from all statistical analyses. 


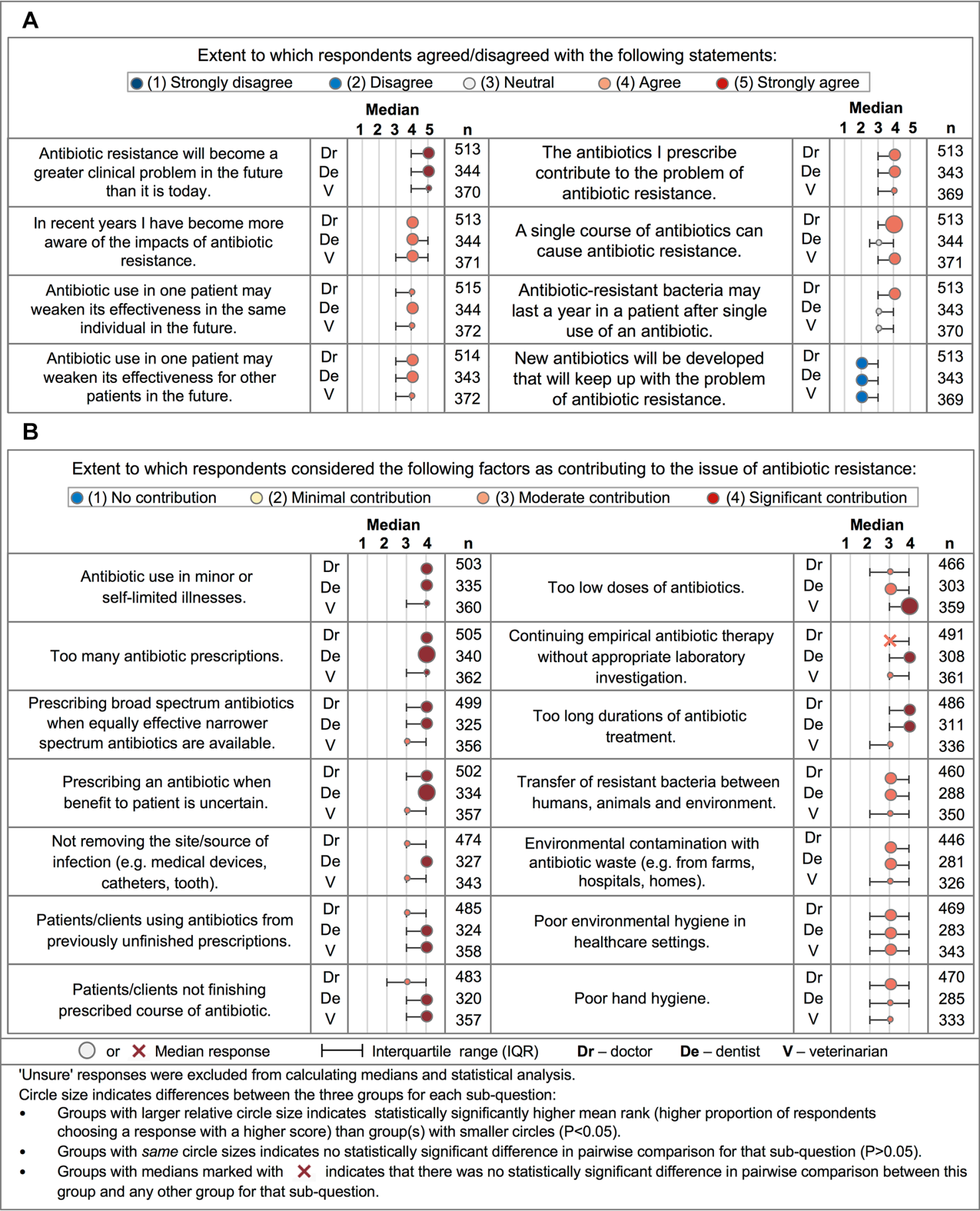

Figure 1 (A,B) Respondents' knowledge and perceptions of antibiotic effectiveness and causes of AbR. (A) Knowledge and beliefs about $A b R$ and $(B)$ perceptions of factors contributing to the issue of $A b R$. AbR, antibiotic resistance.

\section{RESULTS}

\section{Sample characteristics}

Overall, 1549 professionals responded to the survey. However, 219 were excluded because their responses to demographic questions were incomplete, leaving 1330 responses comprising 547 doctors, 380 dentists and 403 veterinarians, representing $0.6 \%, 2.3 \%$ and $3.9 \%$, respectively, of each national workforce (table 1). Respondents were broadly comparable to the characteristics of the national workforce of the three prescriber groups across
Australian states and territories although there were some anomalies (eg, Victoria doctors were under-represented, Western Australia dentists were over-represented and Queensland veterinarians were under-represented (table 1)).

For doctors and dentists, about equal numbers of women and men responded. Among veterinarians, women were over-represented (table 1). Respondent age profiles in all three profession samples were broadly consistent with the national age profile of each profession. 


\begin{tabular}{|c|c|c|c|c|c|c|c|c|c|c|c|c|}
\hline \multicolumn{5}{|c|}{ A } & \multicolumn{4}{|l|}{ B } & \multicolumn{4}{|l|}{ C } \\
\hline & \multicolumn{4}{|c|}{$\begin{array}{l}\text { Extent to which respondents believed } \\
\text { that current levels of antibiotic use in } \\
\text { the following as contributing to the } \\
\text { issue of antibioticic resistance: }\end{array}$} & \multicolumn{4}{|c|}{$\begin{array}{l}\text { Extent to which respondents } \\
\text { believed that antibiotic resistance is a } \\
\text { problem to the health of the } \\
\text { following: }\end{array}$} & \multicolumn{4}{|c|}{$\begin{array}{l}\text { Perceived importance of the roles of } \\
\text { each of the following in } \\
\text { managing/preventing the issue of } \\
\text { antibiotic resistance: }\end{array}$} \\
\hline & \multicolumn{4}{|c|}{\begin{tabular}{|l|} 
(1) No contribution \\
(2) Minimal contribution \\
(3) Moderate contribution \\
(4) Significant contribution \\
\end{tabular}} & \multicolumn{3}{|c|}{\begin{tabular}{|l|} 
(1) Not a problem \\
(2) Minor problem \\
(3) Moderate problem \\
(4) Significant problem \\
\end{tabular}} & & \multicolumn{3}{|c|}{$\begin{array}{l}\text { (1) Not important } \\
\text { (2) Somewhat important } \\
\text { (3) Moderately important } \\
\text { (4) Very important } \\
\text { (5) Extremely important }\end{array}$} & \\
\hline & \multicolumn{4}{|c|}{$\begin{array}{l}\text { Median } \\
1234 \mathrm{n} \\
\end{array}$} & \multicolumn{3}{|r|}{$\begin{array}{l}\text { Median } \\
1234 \\
\end{array}$} & n & \multicolumn{4}{|c|}{$\begin{array}{c}\text { Median } \\
12345 \mathrm{n}\end{array}$} \\
\hline \multirow{2}{*}{$\begin{array}{l}\text { Global } \\
\text { scale }\end{array}$} & $\begin{array}{l}\text { Unregulated use } \\
\text { of antibiotics } \\
\text { globally }\end{array}$ & $\mid \begin{array}{c}\mathrm{Dr} \\
\mathrm{De} \\
\mathrm{V}\end{array}$ & : & $\begin{array}{l}473 \\
315 \\
341\end{array}$ & $\begin{array}{l}\text { People in } \\
\text { developing } \\
\text { countries }\end{array}$ & $\begin{array}{ll}\mathrm{Dr} \\
\mathrm{De} \\
\mathrm{V}\end{array}$ & $\underbrace{-\infty}_{0 \rightarrow-1}$ & $\begin{array}{l}474 \\
315 \\
322\end{array}$ & $\begin{array}{c}\text { Global } \\
\text { organisations } \\
\text { (e.g. WHO, OIE) }\end{array}$ & $\begin{array}{c}\mathrm{Dr} \\
\mathrm{De} \\
\mathrm{V}\end{array}$ & $:-1$ & $\begin{array}{l}495 \\
335 \\
360\end{array}$ \\
\hline & & & & & $\begin{array}{l}\text { People in } \\
\text { developed } \\
\text { countries }\end{array}$ & \begin{tabular}{|c|}
$\mathrm{Dr}$ \\
$\mathrm{De}$ \\
$\mathrm{V}$ \\
\end{tabular} & $\stackrel{\mapsto:}{\mapsto}$ & $\begin{array}{l}496 \\
328 \\
344 \\
\end{array}$ & Governments & $\begin{array}{c}\mathrm{Dr} \\
\mathrm{De} \\
\mathrm{V}\end{array}$ & $\stackrel{\bullet-1}{\bullet-1}$ & $\begin{array}{l}497 \\
333 \\
359 \\
\end{array}$ \\
\hline \multirow{6}{*}{$\begin{array}{l}\text { Antibiotic } \\
\text { use in } \\
\text { humans }\end{array}$} & $\begin{array}{l}\text { In human } \\
\text { hospitals }\end{array}$ & \begin{tabular}{|c|}
$\mathrm{Dr}$ \\
$\mathrm{De}$ \\
$\mathrm{V}$ \\
\end{tabular} & $\begin{array}{l}\mapsto: \\
\vdash\end{array}$ & $\begin{array}{l}484 \\
314 \\
313\end{array}$ & $\begin{array}{l}\text { Patients in } \\
\text { human medical } \\
\text { hospitals }\end{array}$ & \begin{tabular}{|c|}
$\mathrm{Dr}$ \\
$\mathrm{De}$ \\
$\mathrm{V}$ \\
\end{tabular} & $\mapsto$ & $\begin{array}{l}499 \\
326 \\
338 \\
\end{array}$ & $\begin{array}{l}\text { Hospital } \\
\text { doctors }\end{array}$ & \begin{tabular}{|c|}
$\mathrm{Dr}$ \\
$\mathrm{De}$ \\
$\mathrm{V}$
\end{tabular} & 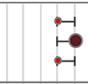 & $\begin{array}{l}499 \\
336 \\
359\end{array}$ \\
\hline & $\begin{array}{l}\text { In nursing } \\
\text { homes/aged care } \\
\text { facilities }\end{array}$ & $\begin{array}{c}\mathrm{Dr} \\
\mathrm{De} \\
\mathrm{V}\end{array}$ & 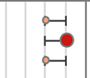 & $\begin{array}{l}447 \\
278 \\
255\end{array}$ & \begin{tabular}{|c|} 
Residents in \\
nursing homelaged \\
care facilities
\end{tabular} & $\begin{array}{l}\mathrm{Dr} \\
\mathrm{De} \\
\mathrm{V}\end{array}$ & 1-9 & \begin{tabular}{|l||}
472 \\
314 \\
309
\end{tabular} & $\begin{array}{l}\text { General medical } \\
\text { practitioners }\end{array}$ & $\begin{array}{c}\mathrm{Dr} \\
\mathrm{De} \\
\mathrm{V}\end{array}$ & $\stackrel{-1}{:-1}$ & $\begin{array}{l}498 \\
335 \\
359\end{array}$ \\
\hline & $\begin{array}{l}\text { In general } \\
\text { medical practice }\end{array}$ & $\begin{array}{c}\mathrm{Dr} \\
\mathrm{De} \\
\mathrm{V}\end{array}$ & $\stackrel{0-1}{-0}$ & $\begin{array}{l}487 \\
320 \\
332\end{array}$ & $\begin{array}{l}\text { The general } \\
\text { public }\end{array}$ & $\begin{array}{l}\mathrm{Dr} \\
\mathrm{De} \\
\mathrm{V}\end{array}$ & Or- & \begin{tabular}{|l|}
490 \\
330 \\
342
\end{tabular} & $\begin{array}{l}\text { The general } \\
\text { public }\end{array}$ & $\begin{array}{c}\mathrm{Dr} \\
\mathrm{De} \\
\mathrm{V}\end{array}$ & $\underset{:-1}{\infty}$ & $\begin{array}{l}499 \\
336 \\
358\end{array}$ \\
\hline & $\begin{array}{l}\text { In human } \\
\text { dental practice }\end{array}$ & \begin{tabular}{|c|}
$\mathrm{Dr}$ \\
$\mathrm{De}$ \\
$\mathrm{V}$
\end{tabular} & $\begin{array}{l}-0 \\
-0 \\
0-1\end{array}$ & $\begin{array}{l}345 \\
328 \\
239 \\
\end{array}$ & $\begin{array}{l}\text { Patients in } \\
\text { human dental } \\
\text { hospitals }\end{array}$ & \begin{tabular}{|c|}
$\mathrm{Dr}$ \\
$\mathrm{De}$ \\
$\mathrm{V}$ \\
\end{tabular} & $\begin{array}{l}\mapsto 0-1 \\
\mapsto-1 \\
\mapsto\end{array}$ & \begin{tabular}{l|}
340 \\
326 \\
254 \\
\end{tabular} & Dentists & $\begin{array}{c}\mathrm{Dr} \\
\mathrm{De} \\
\mathrm{V}\end{array}$ & $\overrightarrow{0}$ & $\begin{array}{l}496 \\
335 \\
359\end{array}$ \\
\hline & & & & & & & & & Pharmacists & $\begin{array}{c}\mathrm{Dr} \\
\mathrm{De} \\
\mathrm{V}\end{array}$ & $\mapsto$ & $\begin{array}{l}498 \\
335 \\
360\end{array}$ \\
\hline & & & & & & & & & Nurses & \begin{tabular}{|c|}
$\mathrm{Dr}$ \\
$\mathrm{De}$ \\
$\mathrm{V}$
\end{tabular} & 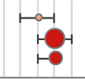 & $\begin{array}{l}497 \\
336 \\
360\end{array}$ \\
\hline \multirow{4}{*}{$\begin{array}{l}\text { Antibiotic } \\
\text { use in } \\
\text { animals }\end{array}$} & In livestock & \begin{tabular}{|c|}
$\mathrm{Dr}$ \\
$\mathrm{De}$ \\
$\mathrm{V}$ \\
\end{tabular} & 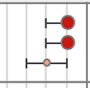 & $\begin{array}{l}378 \\
244 \\
340 \\
\end{array}$ & $\begin{array}{l}\text { Livestock and } \\
\text { food animal } \\
\text { industry }\end{array}$ & \begin{tabular}{|c|}
$\mathrm{Dr}$ \\
$\mathrm{De}$ \\
$\mathrm{V}$
\end{tabular} & 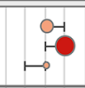 & $\begin{array}{l}250 \\
195 \\
326 \\
\end{array}$ & Veterinarians & $\begin{array}{cc}\mathrm{Dr} \\
\mathrm{De} \\
\mathrm{V}\end{array}$ & $\vec{x}$ & $\begin{array}{l}494 \\
333 \\
360\end{array}$ \\
\hline & In aquaculture & \begin{tabular}{|c|}
$\mathrm{Dr}$ \\
$\mathrm{De}$ \\
$\mathrm{V}$ \\
\end{tabular} & $\begin{array}{c}\mathrm{O}-1 \\
\mathrm{O}-1 \\
\mapsto\end{array}$ & \begin{tabular}{|l|}
306 \\
205 \\
244 \\
\end{tabular} & $\begin{array}{c}\text { Patients in } \\
\text { veterinary clinics }\end{array}$ & $\begin{array}{c}\mathrm{Dr} \\
\mathrm{De} \\
\mathrm{V}\end{array}$ & م-1 & \begin{tabular}{l|}
249 \\
207 \\
357 \\
\end{tabular} & $\begin{array}{l}\text { Farmers and } \\
\text { food producers }\end{array}$ & $\begin{array}{c}\mathrm{Dr} \\
\mathrm{De} \\
\mathrm{V}\end{array}$ & :- & $\begin{array}{l}495 \\
332 \\
359 \\
\end{array}$ \\
\hline & $\begin{array}{l}\text { In companion } \\
\text { animals }\end{array}$ & \begin{tabular}{|c|}
$\mathrm{Dr}$ \\
$\mathrm{De}$ \\
$\mathrm{V}$ \\
\end{tabular} & 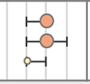 & \begin{tabular}{|l|}
335 \\
219 \\
347 \\
\end{tabular} & Horses & \begin{tabular}{|c|}
$\mathrm{Dr}$ \\
$\mathrm{De}$ \\
$\mathrm{V}$ \\
\end{tabular} & $\begin{array}{l}10 \\
10-1 \\
0-1\end{array}$ & \begin{tabular}{|l|}
210 \\
169 \\
298 \\
\end{tabular} & Pet owners & \begin{tabular}{|c|}
$\mathrm{Dr}$ \\
$\mathrm{De}$ \\
$\mathrm{V}$ \\
\end{tabular} & $\begin{array}{l}\text { ray } \\
\text { ray } \\
\text { ray }\end{array}$ & $\begin{array}{l}492 \\
332 \\
359 \\
\end{array}$ \\
\hline & & & & & Dogs and cats & \begin{tabular}{|c|}
$\mathrm{Dr}$ \\
$\mathrm{De}$ \\
$\mathrm{V}$ \\
\end{tabular} & to- & \begin{tabular}{|l|}
213 \\
169 \\
353 \\
\end{tabular} & & & & \\
\hline \multirow[t]{3}{*}{$\begin{array}{c}\text { My } \\
\text { practice }\end{array}$} & $\begin{array}{l}\text { In my principal } \\
\text { place of practice }\end{array}$ & \begin{tabular}{|l|}
$\mathrm{Dr}$ \\
$\mathrm{De}$ \\
$\mathrm{V}$ \\
\end{tabular} & \begin{tabular}{l|l|} 
& 0 \\
0 \\
$0 \rightarrow 1$
\end{tabular} & \begin{tabular}{|l||}
484 \\
325 \\
355 \\
\end{tabular} & My patients & \begin{tabular}{c|}
$\mathrm{Dr}$ \\
$\mathrm{De}$ \\
$\mathrm{V}$ \\
\end{tabular} & $\begin{array}{l}0-1 \\
0-1 \\
0-1 \\
0-1\end{array}$ & \begin{tabular}{l|}
498 \\
329 \\
361 \\
\end{tabular} & Myself & \begin{tabular}{c|}
$\mathrm{Dr}$ \\
$\mathrm{De}$ \\
$\mathrm{V}$ \\
\end{tabular} & 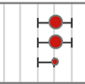 & $\begin{array}{l}499 \\
336 \\
360 \\
\end{array}$ \\
\hline & & & & & Myself & \begin{tabular}{|l}
$\mathrm{Dr}$ \\
$\mathrm{De}$ \\
$\mathrm{V}$ \\
\end{tabular} & $\begin{array}{l}\mapsto 0 \\
\mapsto-1 \\
0-1\end{array}$ & \begin{tabular}{|l|}
492 \\
330 \\
358 \\
\end{tabular} & $\begin{array}{c}\text { My immediate } \\
\text { colleagues/ } \\
\text { peers }\end{array}$ & $\begin{array}{c}\mathrm{Dr} \\
\mathrm{De} \\
\mathrm{V} \\
\end{array}$ & $\stackrel{\mapsto-1}{\mapsto-1}$ & $\begin{array}{l}500 \\
336 \\
360 \\
\end{array}$ \\
\hline & & & & & & & & & $\begin{array}{c}\text { My } \\
\text { clients/patients }\end{array}$ & \begin{tabular}{|c|}
$\mathrm{Dr}$ \\
$\mathrm{De}$ \\
$\mathrm{V}$ \\
\end{tabular} & 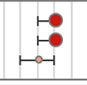 & \begin{tabular}{|l|}
499 \\
335 \\
358 \\
\end{tabular} \\
\hline \multirow[t]{3}{*}{ Other } & & & & & & & & & $\begin{array}{l}\text { Pharmaceutical } \\
\text { companies }\end{array}$ & $\begin{array}{c}\mathrm{Dr} \\
\mathrm{De} \\
\mathrm{V}\end{array}$ & :-1 & $\begin{array}{l}496 \\
333 \\
359 \\
\end{array}$ \\
\hline & & & & & & & & & The Media & $\begin{array}{c}\mathrm{Dr} \\
\mathrm{De} \\
\mathrm{V}\end{array}$ & $\stackrel{-1}{\bullet-1}$ & $\begin{array}{l}498 \\
333 \\
359 \\
\end{array}$ \\
\hline & & & & & & & & & Scientists & \begin{tabular}{|c|}
$\mathrm{Dr}$ \\
$\mathrm{De}$ \\
$\mathrm{V}$ \\
\end{tabular} & $\stackrel{\bullet-1}{\mapsto-1}$ & $\begin{array}{l}496 \\
335 \\
358 \\
\end{array}$ \\
\hline & or $X$ Median & respo & & $\longmapsto$ & Interquartile range (I & (IQR) & Dr-doc & & De - dentist $\mathbf{V}-\mathbf{v}$ & eterinar & & \\
\hline \multicolumn{13}{|c|}{$\begin{array}{l}\text { 'Unsure' responses were excluded from calculating medians and statistical analyses. } \\
\text { Circle size indicates differences between the three groups for each sub--question: } \\
\text { Groups witt larger relative circle size indicates statistically significantly higher mean rank (higher proportion of respondents choosing a } \\
\text { response with a higher score) than group }(s) \text { with smalller circles }(P<0.05 \text { ). } \\
\text { Groups with same circle sizes indicates no statititically significant difference in pairwise comparison for that sub-question ( } P>0.05 \text { ). } \\
\text { Groups with medians marked with } X \text { indicates that there was no statistically significant difference in pairwise comparison between this } \\
\text { group and any other group for that sub-question. }\end{array}$} \\
\hline
\end{tabular}

Figure 2 (A-C) Respondents' perceptions of drivers, extent of the problem and importance of stakeholders in the issue of AbR. (A) Beliefs about current levels of antibiotic use as drivers of AbR, (B) perceived extent of the AbR problem and (C) perceived importance of stakeholders in managing/preventing the issue of AbR. AbR, antibiotic resistance.

Half of the doctors $(52.8 \%)$ and a majority of dentists $(62.4 \%)$ and veterinarians $(77.2 \%$; mostly small companion animal 66.5\%) were in private practice (table 2). Among doctors, the proportion of respondents that worked in each particular setting (eg, private practice and hospital) was comparable with national workforce data (table 2). Dentists working in publicly funded services were over-represented and veterinarians working in mixed practices were under-represented (table 2). 


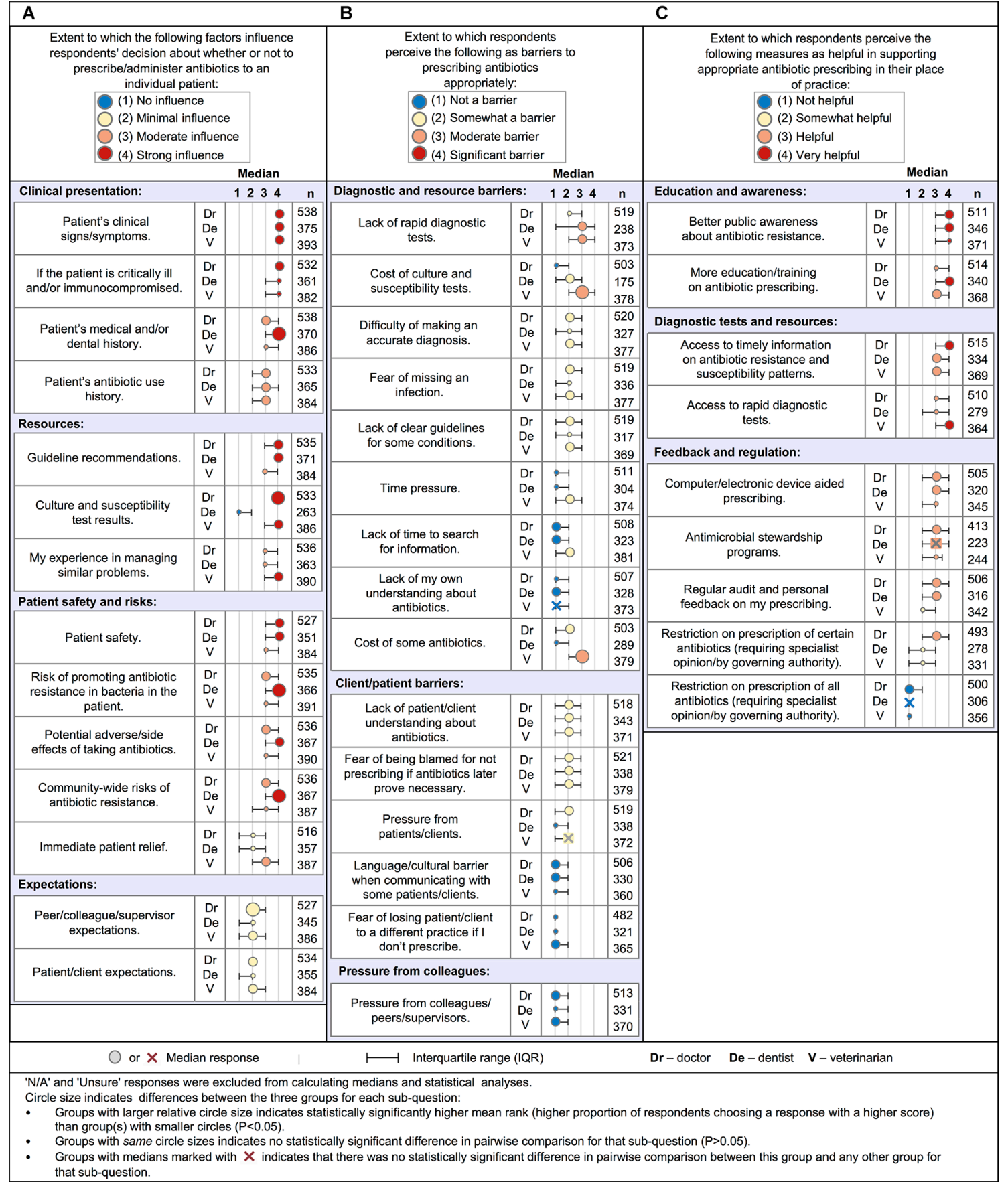

Figure 3 (A-C) Factors influencing prescribing decisions and perceptions of strategies to improve antibiotic prescribing. (A) Factors influencing decisions whether or not to prescribe antibiotics, (B) barriers to prescribing antibiotics appropriately and (C) perceived helpfulness of measures aimed at supporting appropriate prescribing of antibiotics.

\section{Knowledge and perceptions about AbR}

The following results report on the median response categories (in quotation marks) of each prescriber group. Results of the statistical tests, and commonalities and statistically significant differences $(p<0.05)$ between prescriber groups' responses are shown in figures 1-3. See online supplementary material for complete data tables.

There were similarities and differences between doctors, dentists and veterinarians with respect to their levels of knowledge and awareness of AbR (figure 1A).
Dentists were less likely than doctors and veterinarians to agree that a single course of antibiotics can cause AbR (figure 1A). Doctors were more likely than dentists and veterinarians to agree that $\mathrm{AbR}$ bacteria may last a year in a patient after a single use of an antibiotic (figure 1A).

Each prescriber group generally regarded too many antibiotic prescriptions and prescribing for minor or self-limiting illnesses as 'significant' contributors to AbR (figure 1B). There were some differences: veterinarians were less likely than others to rate unnecessary use of broad-spectrum agents, prescribing with uncertain 
Table 1 General characteristics of survey respondents and national doctor, dental and veterinary workforce

\begin{tabular}{|c|c|c|c|c|c|c|c|c|c|c|c|c|}
\hline & \multicolumn{2}{|c|}{$\begin{array}{l}\text { Doctors } \\
\text { (survey) }\end{array}$} & \multicolumn{2}{|c|}{$\begin{array}{l}\text { National } \\
\text { medical } \\
\text { practitioner } \\
\text { workforce* }^{*}\end{array}$} & \multicolumn{2}{|c|}{$\begin{array}{l}\text { Dentists } \\
\text { (survey) }\end{array}$} & \multicolumn{2}{|c|}{$\begin{array}{l}\text { National } \\
\text { dentist } \\
\text { workforce† }\end{array}$} & \multicolumn{2}{|c|}{$\begin{array}{l}\text { Veterinarians } \\
\text { (survey) }\end{array}$} & \multicolumn{2}{|c|}{$\begin{array}{l}\text { National } \\
\text { veterinarian } \\
\text { workforce }\end{array}$} \\
\hline \multicolumn{13}{|c|}{ States/territories } \\
\hline NSW & 163 & 29.8 & 28144 & 32.0 & 72 & 18.9 & 5145 & 31.6 & 111 & 27.5 & 2735 & 26.8 \\
\hline QLD & 114 & 20.8 & 17551 & 19.9 & 111 & 29.2 & 3238 & 19.9 & 37 & 9.2 & 2434 & 23.8 \\
\hline SA & 55 & 10.1 & 6713 & 7.6 & 6 & 1.6 & 1168 & 7.2 & 10 & 2.5 & 616 & 6.0 \\
\hline WA & 46 & 8.4 & 8952 & 10.2 & 131 & 34.5 & 1749 & 10.7 & 93 & 23.1 & 1258 & 12.3 \\
\hline TAS & 20 & 3.7 & 1900 & 2.2 & 9 & 2.4 & 231 & 1.4 & 32 & 7.9 & 219 & 2.1 \\
\hline Total & 547 & 100.0 & 88040 & 100.0 & 380 & 100.0 & 16305 & 100.0 & 403 & 100.0 & 10207 & 100.0 \\
\hline \multicolumn{13}{|l|}{ Gender } \\
\hline Female & 284 & 51.9 & 35282 & 40.1 & 183 & 48.0 & 5452 & 39.0 & 261 & 64.9 & 5513 & 55.9 \\
\hline Male & 263 & 48.1 & 52758 & 59.9 & 197 & 52.0 & 8527 & 61.0 & 141 & 35.1 & 4346 & 44.1 \\
\hline Valid total & 547 & 100.0 & 88040 & 100.0 & 380 & 100.0 & 13979 & 100.0 & 402 & 100.0 & 9860 & 100.0 \\
\hline Missing & 0 & & 0 & & 0 & & 0 & & 1 & & 0 & \\
\hline \multicolumn{13}{|l|}{ Age (years) } \\
\hline$<35$ & 149 & 27.7 & 20344 & 23.1 & 112 & 29.9 & 4462 & 33.7 & 139 & 34.9 & 3512 & 35.6 \\
\hline $35-54$ & 227 & 42.3 & 43774 & 49.7 & 152 & 40.4 & 5935 & 44.8 & 179 & 45.0 & 4684 & 47.5 \\
\hline \multicolumn{13}{|c|}{ Years of experience } \\
\hline $10-19$ & 130 & 23.8 & nd & nd & 84 & 22.2 & nd & nd & 109 & 27.0 & nd & nd \\
\hline $20-29$ & 84 & 15.4 & nd & nd & 60 & 15.8 & nd & nd & 66 & 16.4 & nd & nd \\
\hline$\geq 30$ & 163 & 29.9 & nd & nd & 118 & 31.1 & nd & nd & 94 & 23.3 & nd & nd \\
\hline Valid total & 546 & 100.0 & nd & nd & 379 & 100.0 & nd & nd & 403 & 100.0 & nd & nd \\
\hline Missing & 1 & & & & 1 & & & & 0 & & & \\
\hline
\end{tabular}

${ }^{*}$ National medical workforce data from AlHW. ${ }^{42}$

†National dentist workforce state/territory data from Dental Board of Australia. ${ }^{43}$ Gender and age data from AlHW. ${ }^{44}$ $\ddagger$ National veterinarian workforce data from AVA. ${ }^{45}$

ACT, Australian Capital Territory; AlHW, Australian Institute of Health and Welfare; AVA, Australian Veterinary Association; QLD, Queensland; nd, no data available; NSW, New South Wales; NT, Northern Territory; SA, South Australia; TAS, Tasmania; VIC, Victoria; WA, Western Australia.

benefit and longer antibiotic courses as 'significant', but more likely to rate low doses of antibiotics as 'significant' contributors to ABR; dentists were more likely than others to rate failure to remove the source of infection as 'significant'; and doctors were less likely than others to consider patients not taking a full course or using leftover antibiotics as 'significant' (figure 1B). All groups rated transfer of resistant bacteria between humans, animals and environment, environmental contamination with antibiotic waste and hygiene as 'moderate' contributors (figure 1B).

Each group rated current levels of unregulated use of antibiotics globally and antibiotic use in human hospitals, as making 'significant' contributions to AbR (figure 2A). Dentists rated current levels of antibiotic use in nursing homes/aged care facilities and in general medical 
Table 2 Respondents' fields of work and practice types

\begin{tabular}{ccrl}
\multicolumn{2}{c}{ Survey respondents } & & \multicolumn{2}{c}{ National workforce } \\
$n$ & & N & $\%$
\end{tabular}

\section{Medical respondents ${ }^{*}$}

Main field

\begin{tabular}{|c|c|c|c|c|}
\hline GP & 266 & 48.6 & 28329 & 32.2 \\
\hline Specialist & 128 & 23.4 & 31189 & 35.4 \\
\hline Specialist-in-training & 88 & 16.1 & 15336 & 17.4 \\
\hline Hospital non-specialist & 65 & 11.9 & 9880 & 11.2 \\
\hline Other clinician/non-clinician & 0 & 0.0 & 3306 & 3.8 \\
\hline Total & 547 & 100.0 & 88040 & 100.0 \\
\hline \multicolumn{5}{|l|}{ Main work setting } \\
\hline Private practice & 289 & 52.8 & 41902 & 47.6 \\
\hline Hospital practice & 225 & 41.1 & 38235 & 43.4 \\
\hline Educational facility & 10 & 1.8 & 1876 & 2.1 \\
\hline Other & 23 & 4.2 & 6027 & 6.9 \\
\hline Total & 547 & 100.0 & 88040 & 100.0 \\
\hline \multicolumn{5}{|l|}{ Dental respondents $\dagger$} \\
\hline \multicolumn{5}{|l|}{ Main field } \\
\hline General dentist & 336 & 88.4 & 14635 & 89.8 \\
\hline Specialist dentist & $44 \ddagger$ & $11.6 \neq$ & $1670 \S$ & $10.2 \S$ \\
\hline Total & 380 & 100.0 & 16305 & 100.0 \\
\hline \multicolumn{5}{|l|}{ Main work setting } \\
\hline Private practice & 237 & 62.4 & 10320 & 77.8 \\
\hline $\begin{array}{l}\text { Publicly funded services (public hospital, community } \\
\text { healthcare clinic, Aboriginal health service and defence force) }\end{array}$ & 119 & 31.3 & 1514 & 11.4 \\
\hline Educational facility (university) & 24 & 6.3 & 282 & 2.1 \\
\hline Other/not stated & 0 & 0.0 & 1150 & 8.7 \\
\hline Total & 380 & 100.0 & 13266 & 100.0 \\
\hline \multicolumn{5}{|l|}{ Veterinary respondents } \\
\hline \multicolumn{5}{|l|}{ Main field } \\
\hline Non-specialist veterinarian & 361 & 89.6 & 12213 & 96.4 \\
\hline Specialist veterinarian & 42 & 10.4 & 453 & 3.6 \\
\hline Total & 403 & 100.0 & 12666 & 100.0 \\
\hline \multicolumn{5}{|l|}{ Main work setting } \\
\hline Private practice & 311 & 77.2 & 8273 & 81.0 \\
\hline University teaching hospital & 22 & 5.5 & nd & nd \\
\hline Teaching/research & 21 & 5.2 & 635 & 6.2 \\
\hline Not-for-profit veterinary practice (eg, shelter and zoo) & 14 & 3.4 & nd & nd \\
\hline Government & 26 & 6.5 & 488 & 4.8 \\
\hline Industry & 9 & 2.2 & 252 & 2.5 \\
\hline Other & 0 & 0.0 & 559 & 5.5 \\
\hline Total & 403 & 100.0 & 10207 & 100.0 \\
\hline \multicolumn{5}{|l|}{ Animal practice type } \\
\hline $\begin{array}{l}\text { Small companion animal (includes dogs, cats and pocket } \\
\text { pets) }\end{array}$ & 268 & 66.5 & 5314 & 64.2 \\
\hline Equine & 19 & 4.7 & 473 & 5.7 \\
\hline
\end{tabular}

Continued 


\begin{tabular}{|c|c|c|c|c|}
\hline & \multicolumn{2}{|c|}{ Survey respondents } & \multicolumn{2}{|c|}{ National workforce } \\
\hline & $\mathbf{n}$ & $\%$ & $\mathbf{N}$ & $\%$ \\
\hline Livestock (includes beef, dairy, goats, sheep, pigs and poultry) & 30 & 7.5 & 352 & 4.3 \\
\hline Other animals & 11 & 2.7 & 0 & 0.0 \\
\hline No animal handling & 8 & 2.0 & 0 & 0.0 \\
\hline
\end{tabular}

*National medical workforce data from AlHW. ${ }^{42}$ In survey, 'Hospital non-specialist' includes interns. In national workforce statistics, 'Hospital non-specialist' excludes interns.

†National dentist workforce main field data from Dental Board of Australia. ${ }^{43}$ Main work setting data from AlHW. ${ }^{46}$

$\ddagger$ Number of respondents that completed a Dental Board approved specialty.

$\S N u m b e r$ of registered specialist dentists.

INational veterinarian workforce main field data from AVBC (main field registration numbers retrieved on 18 October 2016 ). ${ }^{47}$ Specialist veterinarian registration numbers derived from State Practitioner Board annual reports and registers. Main work setting and animal practice type data from AVA. ${ }^{45}$

AlHW, Australian Institute of Health and Welfare; AVA, Australian Veterinary Association; AVBC, Australasian Veterinary Boards Council; GP, general practitioner; nd, no data available.

practice as 'significant' whereas veterinarians and doctors rated these as making 'moderate' contributions.

Many respondents were 'unsure' of the contributions of antibiotic use in other sectors in AbR (see online supplementary table 4). Among valid responses, doctors and dentists were more likely to perceive antibiotic use in livestock and companion animals as 'significant' and 'moderate', respectively, whereas veterinarians rated these as making 'moderate' and 'minimal' contributions to $\mathrm{AbR}$, respectively (figure $2 \mathrm{~A}$ ).

Doctors generally rated current levels of antibiotic use in 'my principal place of practice' as making a 'moderate' contribution to AbR, whereas dentists and veterinarians perceived prescribing in 'my principal place of practice' as making 'minimal' contributions to AbR (figure 2A).

All prescriber groups rated AbR as a 'significant' problem to the health of patients in human hospitals and residents in nursing homes/aged care facilities (figure 2B). Veterinarians generally rated AbR as a 'moderate' problem for health of the livestock and food animal industry and a 'minor' problem in veterinary patients, including horses, dogs and cats (figure 2B).

Most respondents in each prescriber group perceived that hospital doctors, general practitioners and global organisations, such as the WHO, have 'very' to 'extremely' important roles in preventing and managing ABR (figure 2C). Compared with veterinarians, doctors and dentists were more likely to rate their own (myself) roles as 'very important' in preventing or managing AbR (figure 2C). However, many doctors and veterinarians did not perceive their own role was as important as that of others in their respective prescriber groups (ie, hospital doctors, general practitioners and veterinarians in general) (figure 2C). Dentists' ratings of their own role and roles of their immediate colleagues and dentists in general were congruent ('very' important).

\section{Perceived influence of factors and barriers in prescribing decisions and helpfulness of interventions}

All groups generally reported that patients' clinical signs/ symptoms strongly influenced their prescribing decisions. A patient's condition as critically ill or immunocompromised was rated a 'strong influence' among all groups, especially doctors (figure 3A). Bacterial culture and susceptibility test results were rated as a 'strong influence' on doctors' and veterinarians' decisions to prescribe antibiotics or not, and as exerting 'no influence' or being 'not applicable' among dentists (figure 3A; online supplementary table 7). Veterinarians were more likely than doctors and dentists to rate immediate patient relief as an influence on prescribing decisions.

Factors rated as 'somewhat' of a barrier and were common across the professions included: lack of patient/ client understanding about antibiotics, fear of blame for failure to prescribe if antibiotics were later proven to be necessary, difficulty of making an accurate diagnosis, lack of clear guidelines for some conditions and fear of missing an infection (figure 3B). All three prescriber groups generally rated the following as 'not a barrier': language/culture when communicating with patients/ clients (and their carers), pressure from colleagues or supervisors, fear of patients/clients moving to a different practice and their own lack of understanding (figure 3B).

Prescriber group-specific barriers included the following: veterinarians were more likely than doctors and dentists to perceive time pressure as at least 'somewhat' of a barrier, and the cost of culture and susceptibility tests and the cost of some antibiotics to be at least 'moderate' barriers. Doctors and dentists generally perceived these costs as either lesser barriers, that is, 'somewhat' or 'not a barrier' (figure 3B). Veterinarians were more likely than doctors to perceive lack of rapid diagnostic tests as a 'significant' barrier (figure 3B). Doctors were more likely 
than dentists to perceive pressures from patients as at least 'somewhat' of a barrier (figure 3B).

All prescriber groups generally perceived that better public awareness about AbR, timely access to antibiotic susceptibility patterns, access to rapid diagnostic tests, more education/training in prescribing, computer/ electronic aided prescribing and antimicrobial stewardship programmes would be 'helpful' or 'very helpful' in supporting more appropriate prescribing (figure 3C). Veterinarians generally thought that regular auditing and feedback on prescribing practices would be 'somewhat helpful' whereas doctors and dentists perceived these would be at least 'helpful' (figure 3C). Doctors were more likely than dentists and veterinarians to perceive that restrictions on prescribing of certain antibiotics would be 'helpful' in supporting more appropriate prescribing while all prescriber groups generally perceived that requiring all antibiotics to be authorised by a specialist or government authority would be 'not helpful' (figure 3C).

\section{DISCUSSION}

\section{Principal findings and comparison with other studies}

The results revealed similarities and differences in knowledge, attitudes and perceptions, between prescriber groups, about the importance, risks and causes of AbR, and the extent to which factors and barriers drive antibiotic prescribing decisions. The results also reveal a tendency to externalise responsibility for poor prescribing to 'others' and that there are common and context specific barriers to optimal prescribing.

Prescribers across all groups demonstrated high levels of awareness that AbR will be a greater problem in the future, and of its causes and extent of impact. Respondents' high levels of recognition that the transfer of resistant bacteria between human, animals and environment has a role in the issue of $\mathrm{AbR}$ and that drivers and impacts of AbR exist in multiple contexts suggests that they have an awareness of AbR as a One Health issue and understand the need for policies that target across human, animal and environmental sectors which is in line with the current focus of WHO activities. In addition, all prescriber groups concurred that a range of stakeholders including governments, health and medical practitioners and other experts, and patients/clients at the global to local scales across human and animal settings should have important roles to play in preventing and managing AbR. Most respondents demonstrated an understanding of shared responsibility through acknowledging their own (individual) and own profession's responsibility for mitigating and managing AbR.

Results also revealed some differences between groups; doctors were generally more aware than dentists or veterinarians that a single course of antibiotics could lead to resistance and that resistant bacteria may persist for up to year in a patient after a single use of antibiotics. These responses likely reflect the prescribers' context, where doctors more regularly encounter AbR infections and their immediate consequences, and strong evidence that AbR bacteria and long-term changes in intestinal microbiota can persist for a year after a single course. ${ }^{23}$ There is little comparable evidence about persistence of $\mathrm{AbR}$ bacteria in animals. Other interprofessional differences in perceptions about drivers of ABR are attributable to differences in their clinical practice and experience. For example, the finding that almost all dentists agreed that failure to remove the source of infection is a major contributor to $\mathrm{AbR}$ is consistent with a high proportion of dental infections being accessible to drainage or tooth removal. ${ }^{24}$ Veterinarians probably were more aware of the risk of $\mathrm{ABR}$ induced by subtherapeutic antibiotic doses, because of widespread concern about their use as growth promoters, based on evidence that exposure of bacteria to low antibiotic concentrations in vitro promotes mutagenesis and horizontal gene transfer. ${ }^{25}$ Veterinarians were less concerned, than others, about the effect on ABR, of broad-spectrum antibiotics or unnecessarily long courses, perhaps because guidelines about choice and duration of therapy, for different conditions in animals are less available than for human use. ${ }^{9}$ Doctors were less concerned about patients not finishing a full course of antibiotic, which is consistent with recent discussion in the medical literature about lack of evidence that it contributes to $\mathrm{ABR}^{2627}$

While most doctors and veterinarians agreed that antibiotic prescribing by their professions contributed significantly to the issue of AbR, they were less likely to believe prescribing in their own (individual) practice contributes to the same extent. Many doctors and dentists, in common with human health practitioners in previous studies, saw antibiotic use in livestock as a significant driver of AbR in humans. ${ }^{21}{ }^{28}$ On the other hand, consistent with some existing studies, many veterinarians did not. ${ }^{29}$ This tension may reflect the limited evidence of, uncertainty about, and geographical variation in contributions of agricultural antibiotic use and environmental contamination to AbR in humans. ${ }^{25}{ }^{30}$ Since most veterinary respondents were small animal veterinarians, they likely do not consider their work as contributing to resistance within the food chain. ${ }^{31}$ Attribution of blame or externalisation of responsibility to others is a major barrier to behavioural change and must be understood, accounted for, and managed in developing policies. ${ }^{32}$ Enabling behavioural change will require that prescribers perceive their own personal roles and actions as efficacious and important in relation to $\mathrm{AbR} .{ }^{33}$ Our results reveal a strong acknowledgement particularly among doctors of their role in helping to manage AbR.

Lack of patient/client understanding of antibiotics, difficulty making an accurate diagnosis and fear of missing an infection or being blamed for poor outcomes were generally rated as barriers to appropriate prescribing, at least to some extent, across all groups. From a policy-making perspective, it is important to recognise these common barriers because they indicate broader challenges across individual professions or settings, such as 
the need for greater public awareness of AbR and a need to reduce diagnostic uncertainty by developing faster and more accessible diagnostic tests to limit the need for risk averse strategies of prescribing unnecessarily or 'just in case' ${ }^{34}$ Sharing of resources, experience, approaches or knowledge across disciplines should help to identify and effect possible solutions. Consistent with the increasing demand for integrated antimicrobial use and AbR surveillance across human and animal health, ${ }^{35}$ our results suggest that changes in prescribing behaviour could be facilitated through exchanging information across different prescriber groups.

At the same time, specific efforts will need to address profession-specific issues, ensuring efficient resource allocation. ${ }^{36}$ For example, structural and commercial pressures were greater for veterinarians than dentists and doctors in the sample, highlighting the potential need for targeted approaches. While solutions exist for doctors and dentists that reduce these barriers, for example, bacterial culture and susceptibility tests in Australia are often publicly subsidised for human patients, there is no such subsidy in the veterinary sector. Comparisons across these prescriber groups reveal important gaps and disparities in resources, levels of knowledge and abilities to commit to best practice. These gaps indicate where there might be room for sharing of resources, such as diagnostic tools and knowledge, policy mechanisms and approaches, across sectors.

Contrary to the existing literature, respondents did not consider commonly reported barriers, such as patient/ client pressure and expectations as having a 'significant' influence on prescribing, despite indicating that better public awareness of the issue would be "very helpful'. ${ }^{37}$ This reveals an inconsistency between respondents perceived and actual experiences. On the one hand, prescribers recognised that they play a role in worsening the problem and did not generally perceive any major barriers to optimal prescribing. On the other, there is substantial evidence of suboptimal antibiotic prescribing in humans and animals. ${ }^{35} 38$ These observations might reflect a limitation of surveys in asking about sensitive topics or undesirable behaviours, but warrant a more sophisticated understanding of the drivers of prescribing behaviour that might be operating.

Prescribers might support interventions that encourage optimal prescribing, including regular auditing and personal feedback. However, they generally perceived more restrictive interventions as 'not helpful'. This implies that the perception of infringement on personal decision-making and professional autonomy is a potential barrier to more restrictive policy-making. ${ }^{39}$ Such views are at odds with a global movement towards formal AbR stewardship programmes and increasingly restrictive policies. ${ }^{40}$ Nevertheless, experience with hospital antimicrobial stewardship programmes in human health suggests that prescribers are responsive to audit, feedback and benchmarking strategies, which support improved prescribing despite diagnostic uncertainties, whereas they are less supportive of outright restrictions. This represents a challenge for policy-making and implementation, which will need to take account of the behaviours of prescribers, their roles as front-line decision-makers, and how they see their rights and responsibilities as experts. Efforts will need to ensure any proposed guidelines, regulations, regular audit cycles and reviews of prescribing practice are not seen as punitive. Policy-makers could look to regulatory approaches in cognate health areas such as tuberculosis and HIV chemotherapies for working examples.

\section{Strengths and limitations of study}

The key strength of the study lies in the simultaneous surveying of doctors, dentists and veterinarians allowing for direct comparisons between knowledge, attitudes and perceptions to reveal the gaps, opportunities and challenges for a One Health approach towards appropriate use of antibiotics. To our knowledge, this is a global first.

Certain prescribers were over-represented and others under-represented in terms of the states and territories and main fields in which they practised, and therefore our sample was not completely representative of each profession. As the sample was self-selecting, it may have led to selection bias of those who were already interested in the topic of AbR and/or those held stronger views. A consequence of asking sensitive questions about prescribing behaviour may have led to reporting of responses they thought were 'appropriate' or socially desirable, especially in relation to factors that influenced their prescribing decisions, rather than representing their actual views or experiences. The questionnaire asked participants to reflect in a straightforward manner and to provide general answers about knowledge, attitudes and behaviours, but these dimensions are in reality, situated in and dependent on contexts. A limitation of self-reporting on factors and barriers as influencing prescribing decisions is that this depends on the levels of awareness and salience of these drivers, which may differ between respondents. Further qualitative research should be undertaken to determine how factors and barriers operate and whether prescribers see any other facets as operating as barriers to optimal prescribing in their practice. Nevertheless, this study provides a baseline from which to broadly compare experiences and identifies the needs of a key subset of Australian human and animal health professionals.

\section{Conclusions and implications for One Health policy-making}

A coordinated One Health response to improving antibiotic use and reducing AbR requires shared awareness, support and commitment, of all stakeholders. The majority of respondents across all prescriber groups were aware of the current and increasing problem of AbR, which is an encouraging foundation towards achieving a One Health approach. However, characterisations of AbR more as a human health problem than an animal health problem and externalisation of responsibility of AbR on to others (by all prescriber groups) represent a challenge to achieving a One Health approach, as individuals may 
be less critical of their own actions in relation to others. We suggest that policy-makers and practitioners should look to successes of interventions that have increased doctors' awareness of personal responsibility and adapt and trial these approaches when designing similar interventions for dentists and veterinarians. ${ }^{41}$ Comparisons across groups revealed that different prescribers have specific knowledge gaps and practical uncertainties and are influenced by different sets of factors and barriers when prescribing antibiotics, suggesting that policy-making which includes strategies to improve antibiotic use should be sensitive and tailored to these experiences. Prescribers also have different preferences for and experiences with strategies aimed at supporting appropriate prescribing. However, some barriers and perceptions of strategies are similar across prescribers which suggests that there are opportunities towards achieving a One Health approach to improving antibiotic prescribing and addressing gaps through sharing knowledge and resources across settings and fields. Additionally, design of more restrictive policies should consider the perceived impacts on prescriber autonomy within the bounds of optimal prescribing practice. Although this study focused on prescribers in Australia, we believe that the results are of global relevance.

\section{Author affiliations}

${ }^{1}$ School of Geosciences, The University of Sydney, Sydney, NSW, Australia

${ }^{2}$ School of Life Sciences, University of Technology Sydney, Sydney, New South Wales, Australia

${ }^{3}$ The ithree Institute, University of Technology Sydney, Sydney, New South Wales, Australia

${ }^{4}$ Sydney School of Veterinary Science, University of Sydney, Sydney, New South

Wales, Australia

${ }^{5}$ Marie Bashir Institute for Infectious Diseases and Biosecurity, University of Sydney, Sydney, New South Wales, Australia

${ }^{6}$ Graduate School of Health, University of Technology Sydney, Sydney, New South Wales, Australia

${ }^{7}$ Faculty of Social Science, University of Wollongong, Wollongong, NSW, Australia ${ }^{8}$ Menzies Centre for Health Policy, Sydney School of Public Health, University of Sydney, Sydney, New South Wales, Australia

${ }^{9}$ The Australian Prevention Partnership Centre, The Sax Institute, Sydney, New South Wales, Australia

${ }^{10}$ Sydney Health Ethics, University of Sydney, Sydney, New South Wales, Australia

${ }^{11}$ Faculty of Dentistry, University of Sydney, Sydney, New South Wales, Australia

${ }^{12}$ Institute of Dental Research, Westmead Centre for Oral Health, Sydney, New South Wales, Australia

${ }^{13}$ Sydney School of Public Health, University of Sydney, Sydney, New South Wales, Australia

${ }^{14}$ Westmead Institute for Medical Research, Sydney, New South Wales, Australia

${ }^{15}$ Discipline of Child and Adolescent Health, Sydney Medical School, University of Sydney, Sydney, New South Wales, Australia

${ }^{16}$ Infectious Disease and Microbiology, The Children's Hospital at Westmead,

Sydney, NSW, Australia

${ }^{17}$ Westmead Hospital, Sydney, New South Wales, Australia

Acknowledgements We thank the Faculty of Science and the Marie Bashir Institute for Infectious Diseases and Biosecurity at the University of Sydney for internal grants to support this work. We thank the Office of Health Protection, Australian Government Department of Health for providing feedback on questionnaire design and for assistance in circulating the online survey. We also thank the professional associations, organisations and individuals for their assistance in circulating the online survey, as well as the doctors, dentists and veterinarians who participated in the survey.
Contributors DD-H and ML conceived the study. AZ, DD-H, ML, GLG, JMN, MPW, BVB, K-AN, MG, CD, GAH-C, TCS, JRI, AMK and AD contributed to study design including questionnaire design and development and literature search. AZ, JMN, MG, MPW, BVB, TCS and KA-N contributed to data collection. AZ, MPW and BVB contributed to statistical data analysis. All the authors participated in interpretation of the data, reviewed and commented on the manuscript. DD-H and ML wrote the manuscript.

Funding This study was funded by grants from the Faculty of Science and the Marie Bashir Institute for Infectious Diseases and Biosecurity at the University of Sydney.

Competing interests None declared.

Patient consent Not required.

Ethics approval Ethics approval was granted by the University of Sydney Human Research Ethics Committee (project no: 2016/675).

Provenance and peer review Not commissioned; externally peer reviewed.

Data sharing statement Further data generated by the study and referred to in the manuscript is available via request to the corresponding author.

Open Access This is an Open Access article distributed in accordance with the Creative Commons Attribution Non Commercial (CC BY-NC 4.0) license, which permits others to distribute, remix, adapt, build upon this work non-commercially, and license their derivative works on different terms, provided the original work is properly cited and the use is non-commercial. See: http://creativecommons.org/ licenses/by-nc/4.0/

(C) Article author(s) (or their employer(s) unless otherwise stated in the text of the article) 2018. All rights reserved. No commercial use is permitted unless otherwise expressly granted.

\section{REFERENCES}

1. Laxminarayan R, Duse A, Wattal C, et al. Antibiotic resistance-the need for global solutions. Lancet Infect Dis 2013;13:1057-98.

2. The World Health Organisation (WHO). Global action plan on antimicrobial resistance. 2015 http://www.who.int/antimicrobialresistance/publications/global-action-plan/en/ (accessed $20 \mathrm{Apr}$ 2017).

3. Australian Government. Responding to the threat of antimicrobial resistance - Australia's First National Antimicrobial Resistance Strategy 2015-2019. 2015 http:// www.health.gov.au/internet/main/publishing.nsf/Content/ 1803C433C71415CACA257C8400121B1F/\%24File/amr-strategy2015-2019.pdf (accessed 20 Apr 20 2016).

4. King LJ. Combating the Triple Threat: The Need for a One Health Approach. Microbiol Spectr 2013;1.

5. Holmes AH, Moore LS, Sundsfjord A, et al. Understanding the mechanisms and drivers of antimicrobial resistance. Lancet 2016;387:176-87.

6. Arnold SR, Straus SE. Interventions to improve antibiotic prescribing practices in ambulatory care. Cochrane Database Syst Rev 2005;4:CD003539.

7. Hulscher ME, Grol RP, van der Meer JW. Antibiotic prescribing in hospitals: a social and behavioural scientific approach. Lancet Infect Dis 2010;10:167-75.

8. Newlands R, Duncan EM, Prior M, et al. Barriers and facilitators of evidence-based management of patients with bacterial infections among general dental practitioners: a theory-informed interview study. Implement Sci 2016;11:1-12.

9. Mateus AL, Brodbelt DC, Barber N, et al. Qualitative study of factors associated with antimicrobial usage in seven small animal veterinary practices in the UK. Prev Vet Med 2014;117:68-78.

10. Md Rezal RS, Hassali MA, Alrasheedy AA, et al. Physicians' knowledge, perceptions and behaviour towards antibiotic prescribing: a systematic review of the literature. Expert Rev Anti Infect Ther 2015;13:665-80.

11. Vandeweerd JM, Vandeweerd S, Gustin C, et al. Understanding veterinary practitioners' decision-making process: implications for veterinary medical education. J Vet Med Educ 2012;39:142-51.

12. De Briyne N, Atkinson J, Pokludová L, et al. Factors influencing antibiotic prescribing habits and use of sensitivity testing amongst veterinarians in Europe. Vet Rec 2013;173:475.

13. D'Angeli MA, Baker JB, Call DR, et al. Antimicrobial stewardship through a one health lens. International Journal of Health Governance 2016;21:114-30. 
14. The World Health Organisation (WHO). Antibiotic resistance. 2016 http://www.who.int/mediacentre/factsheets/antibiotic-resistance/en/ (accessed 8 Mar 2017).

15. Srinivasan A, Song X, Richards A, et al. A survey of knowledge, attitudes, and beliefs of house staff physicians from various specialties concerning antimicrobial use and resistance. Arch Intern Med 2004;164:1451-6.

16. García C, Llamocca LP, García K, et al. Knowledge, attitudes and practice survey about antimicrobial resistance and prescribing among physicians in a hospital setting in Lima, Peru. BMC Clin Pharmacol 2011;11:18.

17. Pulcini C, Williams F, Molinari N, et al. Junior doctors' knowledge and perceptions of antibiotic resistance and prescribing: a survey in France and Scotland. Clin Microbiol Infect 2011;17:80-7.

18. Jacob ME, Hoppin JA, Steers N, et al. Opinions of clinical veterinarians at a US veterinary teaching hospital regarding antimicrobial use and antimicrobial-resistant infections. J Am Vet Med Assoc 2015;247:938-44.

19. Gibbons JF, Boland F, Buckley JF, et al. Influences on antimicrobial prescribing behaviour of veterinary practitioners in cattle practice in Ireland. Vet Rec 2013;172:14.

20. Wood F, Phillips C, Brookes-Howell L, et al. Primary care clinicians perceptions of antibiotic resistance: a multi-country qualitative interview study. J Antimicrob Chemother 2013;68:237-43.

21. Cope AL, Wood F, Francis NA, et al. General dental practitioners' perceptions of antimicrobial use and resistance: a qualitative interview study. Br Dent J 2014;217:E9.

22. Dunn OJ. Multiple Comparisons Using Rank Sums. Technometrics 1964:6:241-52.

23. Costelloe C, Metcalfe C, Lovering A, et al. Effect of antibiotic prescribing in primary care on antimicrobial resistance in individual patients: systematic review and meta-analysis. BMJ 2010;340:c2096.

24. Al-Haroni M. Bacterial resistance and the dental professionals' role to halt the problem. J Dent 2008;36:95-103.

25. Andersson DI, Hughes D. Microbiological effects of sublethal levels of antibiotics. Nat Rev Microbiol 2014;12:465-78.

26. Havey TC, Fowler RA, Daneman N. Duration of antibiotic therapy for bacteremia: a systematic review and meta-analysis. Crit Care 2011;15:R267-R67.

27. Gilbert GL. Knowing when to stop antibiotic therapy. Med J Aust 2015;202:121-2.

28. Giblin TB, Sinkowitz-Cochran RL, Harris PL, et al. Clinicians' perceptions of the problem of antimicrobial resistance in health care facilities. Arch Intern Med 2004;164:1662-8.

29. Jones PJ, Marier EA, Tranter RB, et al. Factors affecting dairy farmers' attitudes towards antimicrobial medicine usage in cattle in England and Wales. Prev Vet Med 2015;121:30-40.

30. Chang Q, Wang W, Regev-Yochay G, et al. Antibiotics in agriculture and the risk to human health: how worried should we be? Evol Appl 2015;8:240-7.

31. Van Boeckel TP, Brower C, Gilbert M, et al. Global trends in antimicrobial use in food animals. Proc Natl Acad Sci U S A 2015;112:5649-54.
32. Rüegg SR, McMahon BJ, Häsler B, et al. A Blueprint to Evaluate One Health. Front Public Health 2017;5:20.

33. Fishbein M, Cappella JN. The Role of Theory in Developing Effective Health Communications. J Commun 2006;56:S1-S17.

34. Belongia EA, Schwartz B. Strategies for promoting judicious use of antibiotics by doctors and patients. BMJ 1998;317:668-71.

35. Shaban RZ, Simon GI, Trott DJ, et al. Surveillance and reporting of antimicrobial resistance and antibiotic usage in animals and agriculture in Australia. 2014 http://www.agriculture.gov.au/ SiteCollectionDocuments/animal-plant/animal-health/amria.pdf (accessed 30 Jun 2017).

36. Dar OA, Hasan R, Schlundt J, et al. Exploring the evidence base for national and regional policy interventions to combat resistance. Lancet 2016;387:285-95.

37. Fletcher-Lartey S, Yee M, Gaarslev C, et al. Why do general practitioners prescribe antibiotics for upper respiratory tract infections to meet patient expectations: a mixed methods study. BMJ Open 2016;6:e012244.

38. Australian Commission on Safety and Quality in Health Care (ACSQHC). AURA 2016: first Australian report on antimicrobial use and resistance in human health. $2016 \mathrm{https} / / \mathrm{www}$.safetyandquality. gov.au/wp-content/uploads/2017/01/AURA-2016-First-AustralianReport-on-Antimicrobial-use-and-resistance-in-human-health.pdf (accessed 30 Jun 2016)

39. Garau J. Impact of antibiotic restrictions: the ethical perspective. Clin Microbiol Infect 2006;12(Suppl 5):16-24.

40. Davey P, Marwick CA, Scott CL, et al. Interventions to improve antibiotic prescribing practices for hospital inpatients. Cochrane Database Syst Rev 2017;2:CD003543.

41. Bal AM, Gould IM. Antibiotic stewardship: overcoming implementation barriers. Curr Opin Infect Dis 2011;24:357-62.

42. Australian Institute of Health and Welfare (AlHW). National Health Workforce Data Set: medical practitioners 2015. 2016 https://www. aihw.gov.au/reports/workforce/medical-practitioners-workforce2015/data (accessed 8 May 2017).

43. Dental Board of Australia. Number of registered dentists in Australia. 2016 http://www.dentalboard.gov.au/About-the-Board/Statistics. aspx.

44. Australian Institute of Health and Welfare (AlHW). Employed dentists in Australia 2014. 2016 http://webarchive.nla.gov.au/ gov/20170404183122/http://www.aihw.gov.au/workforce/dentist/ (accessed 8 May 2017)

45. Australian Veterinary Association (AVA). Australian veterinary workforce modelling. 2015 http://www.ava.com.au/sites/default/files/ AVA_website/pdfs/Australian veterinary workforce modelling report. pdf (accessed 15 May 2017).

46. Australian Institute of Health and Welfare (AlHW). Dental workforce 2012. National health workforce series no 7. 2014 https://www.aihw. gov.au/getmedia/cd0bd5ad-ecd9-40f9-8430-c2818b80e7dd/16428. pdf.aspx?inline=true (accessed 8 May 2017).

47. The Australasian Veterinary Boards Council Inc (AVBC). Search for Registered Veterinarians in Australia. 2016 https://avbc.asn.au/ search-for-registered-vet/ (accessed 18 Oct 2016). 